\title{
Imprinting Vortices into Antiferromagnets
}

\author{
J. Sort, ${ }^{1, *}$ K. S. Buchanan, ${ }^{2, \dagger}$ V. Novosad, ${ }^{2}$ A. Hoffmann, ${ }^{2}$ G. Salazar-Alvarez, ${ }^{3}$ A. Bollero, ${ }^{4}$ \\ M. D. Baró, ${ }^{3}$ B. Dieny, ${ }^{4}$ and J. Nogués ${ }^{1}$ \\ ${ }^{1}$ Institució Catalana de Recerca i Estudis Avançats (ICREA) and Departament de Física, Universitat Autònoma de Barcelona, \\ 08193 Bellaterra, Barcelona, Spain \\ ${ }^{2}$ Materials Science Division and Center for Nanoscale Materials, Argonne National Laboratory, Argonne, Illinois 60439, USA \\ ${ }^{3}$ Departament de Física, Universitat Autònoma de Barcelona, 08193 Bellaterra, Spain \\ ${ }^{4}$ SPINTEC (URA 2512 CNRS/CEA), CEA/Grenoble, 17 Rue des Martyrs, 38054 Grenoble Cedex 9, France
}

(Received 7 April 2006; published 7 August 2006)

\begin{abstract}
The effect of imprinting symmetric and displaced vortex structures into an antiferromagnetic material is investigated in micron-sized disks consisting of exchange coupled ferromagnetic-antiferromagnetic bilayers. The imprint of displaced vortices manifests itself by the occurrence of a new type of asymmetric hysteresis loops characterized by curved, reversible, central sections with nonzero remanent magnetization. Such an imprint is achieved by cooling the disks through the blocking temperature of the system in small fields. Micromagnetic simulations reveal that asymmetric vortexlike loops naturally result from the competition between the different energies involved in the system.
\end{abstract}

PACS numbers: 75.60.Jk, 75.50.Ee, 75.70.Cn

In ferromagnets and antiferromagnets, there are often domains with differently ordered spin states observed instead of a single homogeneously long-range ordered region. It turns out that domain structures in antiferromagnetic (AFM) materials have been far less studied than domains in ferromagnets [1]. This is partially due to the fact that the staggered AFM spin structure with its lack of a net magnetization makes the detection of domains and domain walls more challenging [2,3]. Moreover, the physical properties of AFM domains differ significantly from ferromagnetic (FM) domains. Domains in a ferromagnet are generally determined by balancing magnetostatic with exchange and anisotropy energies. Thus, in nanostructured systems, ferromagnets can develop welldefined unusual spin states, such as magnetic vortices [49]. The absence of a net magnetization in antiferromagnets means that magnetostatic energy is vanishing, and, therefore, domains in antiferromagnets are generally metastable, since typically the gain in configurational entropy is not sufficient to overcome domain wall energies. Without any direct explicit driver for domain formation, the AFM domains may originate from random nucleation of long-range order due to randomly distributed defects [10]. For these reasons, the formation and evolution of AFM domains remain a research area with many open questions.

Nevertheless, domains in antiferromagnets have received increased attention recently due to their role in exchange bias, i.e., the shift of the hysteresis loop observed in FM-AFM coupled systems [11,12], which is a key ingredient in spintronic devices [13]. Many exchange biased systems exhibit unusual properties, one of which is a pronounced asymmetry in the hysteresis loop, caused by different irreversible reversal mechanisms on either side of the hysteresis loop [14-18]. Since the ferromagnet and the antiferromagnet are exchange coupled, measurements of the FM behavior provide an indirect probe of the properties of the antiferromagnet, for example, the spin-flop field [19], the surface order parameter [20], the crystalline anisotropy [21], or the domain wall width [22,23]. Furthermore, it has recently been demonstrated that nonuniformly magnetized ferromagnets can modify the antiferromagnetic spin structure in a way that allows tuning of the hysteresis loops over a wide range [24-26].

In this work, we show that it is possible to imprint a welldefined nonuniform magnetization state, such as a magnetic vortex, into the antiferromagnet. Investigations of the role of magnetic vortices in exchange bias systems have begun only recently [27-31]. In previous experiments, we have shown that the interplay between the unidirectional exchange bias and vortex structures can give rise to angular-dependent magnetization reversal, when the exchange bias is established with a saturated ferromagnet [27,31]. In contrast, here we investigate the magnetization reversal of circular disks composed of FM-AFM bilayers after cooling them in unsaturated magnetization states. This allows imprinting symmetric as well as displaced vortex spin structures into the AFM. The resulting hysteresis loops exhibit a new type of asymmetry characterized by a curved, reversible, central part with nonzero remanent magnetization, which can be accurately controlled by varying the cooling field magnitude. This asymmetry originates from the competition between the intradot magnetostatic energy and the FM-AFM exchange energy with the imprinted structure.

Arrays of circular disks $(1 \mu \mathrm{m}$ diameter $)$ were fabricated by $e$-beam lithography and subsequent ion etching of continuous films, previously deposited onto thermally oxidized Si wafers by dc magnetron sputtering, with the compositions $\quad \mathrm{Ta}(5 \mathrm{~nm}) / \mathrm{Py}(12 \mathrm{~nm}) / \operatorname{IrMn}(5 \mathrm{~nm}) / \mathrm{Pt}(2 \mathrm{~nm})$ (where Py, i.e., permalloy, stands for $\mathrm{Ni}_{80} \mathrm{Fe}_{20}$ and is $\mathrm{FM}$, whereas $\operatorname{IrMn}$, i.e., $\operatorname{Ir}_{20} \mathrm{Mn}_{80}$, is $\left.\mathrm{AFM}\right)$ and $\mathrm{Ta}(5 \mathrm{~nm}) /$ 
$\operatorname{Py}(12 \mathrm{~nm}) / \mathrm{Pt}(2 \mathrm{~nm})$. The patterned structures were heated to $550 \mathrm{~K}$ (i.e., above the blocking temperature of the system, $T_{B}=420 \mathrm{~K}$ ) and subsequently cooled to room temperature both in zero field and with in-plane magnetic fields $\mu_{0} H_{\mathrm{FC}}$, with values ranging from 2.5 to $250 \mathrm{mT}$, applied parallel and perpendicular to the direction of measurement (arbitrarily taken as $0^{\circ}$ ). The dots were separated by $2 \mu \mathrm{m}$ to minimize dipolar interactions. Hysteresis loops were recorded at room temperature using a Durham magneto-optics Kerr effect (MOKE) setup and detecting both the longitudinal and transverse magnetization components, i.e., the in-plane magnetization components parallel and perpendicular to the applied magnetic field, respectively. Magnetic force microscopy (MFM) imaging (using a Nanoscope III Digital Instruments, Inc.) was also performed [32].

To interpret the magnetization curves, micromagnetic simulations were performed using a Landau-LifshitzGilbert micromagnetic solver [33]. Typical material parameters for bulk permalloy were used: saturation magnetization $M_{S}=8 \times 10^{-5} \mathrm{~A} / \mathrm{m}$ and exchange stiffness constant $A=1.3 \times 10^{-11} \mathrm{~J} / \mathrm{m}$. The magnetocrystalline anisotropy was neglected. Hysteresis loops were calculated by allowing the disk magnetization state to relax to an equilibrium state in successively applied magnetic fields. The patterned exchange bias is represented by an additional position-dependent pinning field with local magnitude $\mu_{0} H_{E}=9 \mathrm{mT}$ and with the direction defined by calculations of the unbiased vortex state for an applied field equal to $H_{\mathrm{FC}}$. Full hysteresis loops were obtained using cells of $5.4 \times 5.4 \mathrm{~nm}^{2}, 12 \mathrm{~nm}$ thick at a temperature of $300 \mathrm{~K}$. Simulations of the reversible portion of the curve were repeated with smaller cells $\left(3 \times 3 \mathrm{~nm}^{2}, 12 \mathrm{~nm}\right.$ thick) at zero temperature in order to obtain more refined estimates of the remanent magnetization and core trajectories.

The hysteresis loops of Py and Py-IrMn disks after field cooling (FC) along $0^{\circ}$ from $T=550 \mathrm{~K}$ in $\mu_{0} H_{\mathrm{FC}}=$ $250 \mathrm{mT}$ are shown in Fig. 1(a). Both loops are constricted in their central part, as observed for magnetization reversal via nucleation and annihilation of a vortex state [4-9]. For the disks without IrMn, the loop shape is insensitive to the field cooling. In contrast, in the Py-IrMn disks measured along the FC direction, a symmetric vortexlike loop is observed, shifted along the magnetic field axis by $\mu_{0} H_{E}=$ $9 \mathrm{mT}$. For both the biased and unbiased Py, no transverse magnetization signal is detectable. If the Py-IrMn disks are zero-field cooled (ZFC) from $T=550 \mathrm{~K}$, the resulting hysteresis loop becomes closed (i.e., reversible) at its central part [see gray curve in Fig. 1(b)], again without any detectable transverse component. In this case, the nucleation and annihilation fields of the vortex increase by approximately $9 \mathrm{mT}$ with respect to the unbiased Py, an enhancement of the same magnitude as the loop shift observed in the case of field cooling in saturation. This suggests that during ZFC the vortex state is imprinted into the AFM, and, once at room temperature, the coupling with the AFM acts like a $9 \mathrm{mT}$ local effective pinning field with
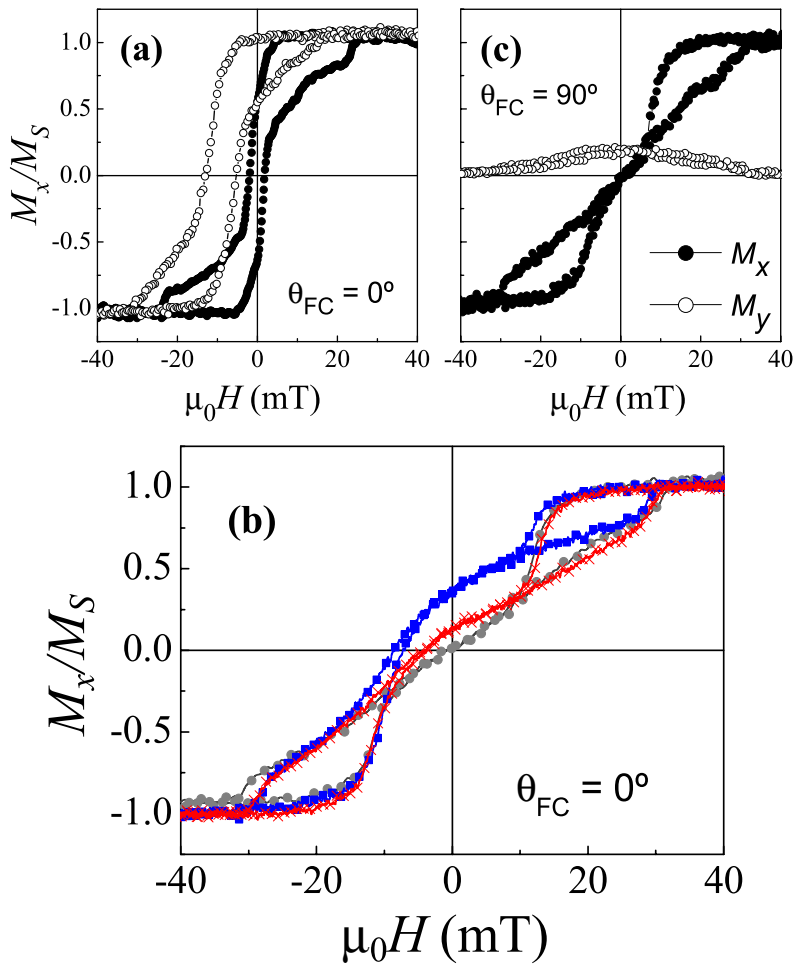

FIG. 1 (color online). Hysteresis loops corresponding to: (a) Py (O) and Py-IrMn $(\bigcirc)$ disks $\mathrm{FC}$ along $\theta_{\mathrm{FC}}=0^{\circ}$ in $\mu_{0} H_{\mathrm{FC}}=250 \mathrm{mT}$; (b) Py-IrMn disks after ZFC (gray ) and $\mathrm{FC}$ in $\mu_{0} H_{\mathrm{FC}}=2.5 \mathrm{mT}\left(\right.$ red $\times$ ) and $\mu_{0} H_{\mathrm{FC}}=10 \mathrm{mT}$ (blue $\left.\mathbf{\square}\right)$ along $\theta_{\mathrm{FC}}=0^{\circ}$; (c) longitudinal $(\bigcirc)$ and transverse $(\bigcirc)$ components of Py-IrMn disks FC along $\theta_{\mathrm{FC}}=90^{\circ}$ in $\mu_{0} H_{\mathrm{FC}}=$ $10 \mathrm{mT}$.

the cylindrical symmetry of a centered vortex state, thus enhancing the magnetic stability of the vortex in the FM.

When the Py-IrMn disks are FC in small $H_{\mathrm{FC}}$ values, the resulting hysteresis loops recorded along the FC direction become asymmetric [Fig. 1(b)]. This asymmetry is evidenced by the appearance of a curved, reversible, central part. The loop curvature and the remanent magnetization increase as $H_{\mathrm{FC}}$ is increased. This allows precise tuning of the loop asymmetry. Similar to the ZFC Py-IrMn disks, no transverse Kerr signal is detected.

On the other hand, when $H_{\mathrm{FC}}$ is applied in-plane but perpendicular $\left(\theta_{\mathrm{FC}}=90^{\circ}\right)$ to the measuring direction, then both longitudinal and transverse magnetization components are observed [Fig. 1(c)]. The asymmetry observed along $\theta_{\mathrm{FC}}=0^{\circ}$ is no longer observed for $\theta_{\mathrm{FC}}=90^{\circ}$, and the remanence in the longitudinal loop is zero. However, the transverse component is now nonzero, indicating that unlike in the ZFC case the vortex core is not centered but displaced perpendicular to the field-cooling direction.

The simulated hysteresis loop for a Py-IrMn disk after ZFC is shown in Fig. 2(a), together with the simulated loop corresponding to Py only. These loops are remarkably similar to their experimental counterparts in Fig. 1 with the exception of the annihilation field, which is slightly larger in the simulated loops. The measured loops are also 

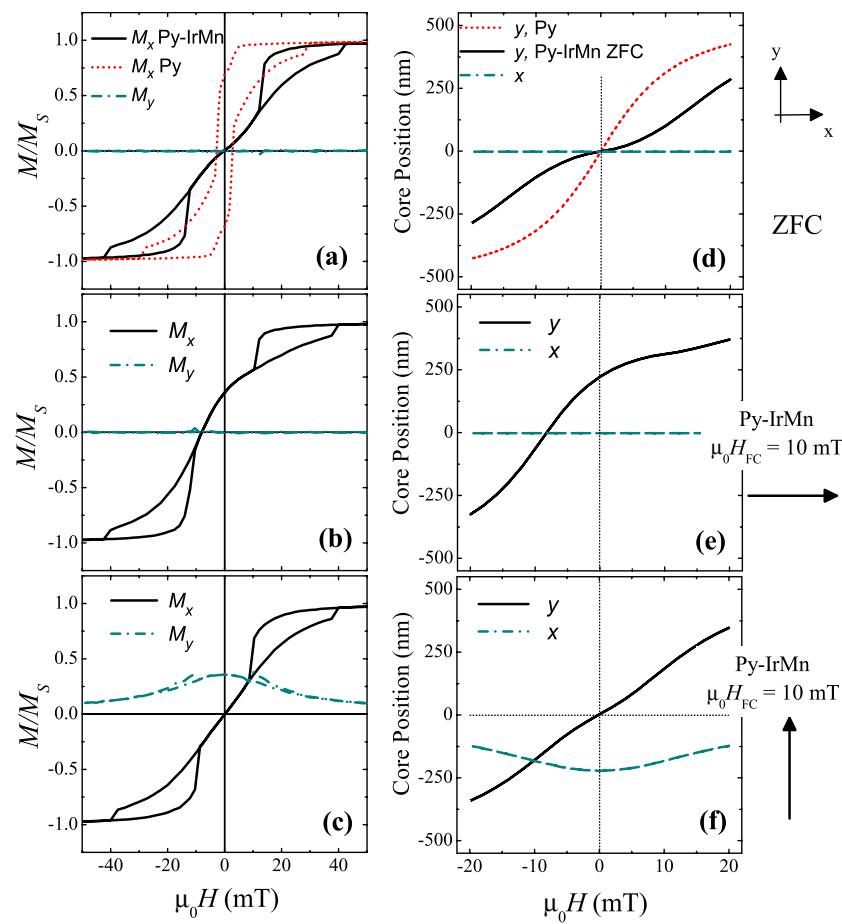

FIG. 2 (color online). (a) Micromagnetic simulations of the hysteresis loops corresponding to Py-IrMn disks (solid line) and Py (dotted line) after ZFC; the micromagnetic simulations predict no transverse signal (dashed-dotted line) for both Py and Py-IrMn; (b) calculated longitudinal (solid line) and transverse (dashed-dotted line) hysteresis loops for $\theta_{\mathrm{FC}}=0^{\circ}$ and $\mu_{0} H_{\mathrm{FC}}=10 \mathrm{mT}$; (c) calculated longitudinal (solid line) and transverse (dashed-dotted line) hysteresis loops for Py-IrMn for $\theta_{\mathrm{FC}}=90^{\circ}$ and $\mu_{0} H_{\mathrm{FC}}=10 \mathrm{mT}$; (d) calculated positions $(x, y)$ of the vortex core center in Py and Py-IrMn ZFC; (e) calculated positions of the vortex core in Py-IrMn, for $\theta_{\mathrm{FC}}=$ $0^{\circ}$ and $\mu_{0} H_{\mathrm{FC}}=10 \mathrm{mT}$; (f) calculated positions of the vortex core in Py-IrMn, for $\theta_{\mathrm{FC}}=90^{\circ}$ and $\mu_{0} H_{\mathrm{FC}}=10 \mathrm{mT}$.

a bit more rounded as compared to the simulated ones. This is probably due to the fact that an ensemble of dots, instead of a single dot, are measured by MOKE, and slight inhomogeneities between the different dots can bring about a distribution of nucleation and annihilation fields. For the ZFC case, the remanence is zero because during the cooling a symmetric vortex state is imprinted to the AFM [as shown schematically in Fig. 3(a)]. During the hysteresis loop, the vortex core moves along a straight line [Fig. 3(e)], perpendicular to the applied field, as revealed by the simulated dependence of the vortex core position as a function of the applied field [Fig. 2(d)]. This process changes the longitudinal magnetization component, but, due to the disk symmetry, the transverse one remains zero, in agreement with the experimental results. The simulations indicate that the magnetic susceptibility in Py-IrMn is smaller than for Py disks, in agreement with the enhanced stability of the vortex state in ZFC Py-IrMn disks.

In contrast to the ZFC case, the simulated longitudinal loop for Py-IrMn FC in $\mu_{0} H_{\mathrm{FC}}=10 \mathrm{mT}$, when applying $H_{\mathrm{FC}}$ along $\theta_{\mathrm{FC}}=0^{\circ}$, reveals a nonzero remanent magne- tization [Fig. 2(b)], resulting from the asymmetric motion of the vortex as a function of field. As before, the core moves only along the $y$ direction, leading to a vanishing transverse magnetization [Fig. 2(e)]. At remanence, the core does not remain at the center of the disk due to the pinning with the imprinted displaced vortex [see Figs. 3(b) and $3(\mathrm{c})$, for $\mu_{0} H_{\mathrm{FC}}=2.5$ and $10 \mathrm{mT}$, respectively] resulting in a nonzero remanent magnetization. Because of the interplay between Zeeman, magnetostatic, and exchange energies, the vortex core is closer to the disk center at remanence as compared to the corresponding pinning field distribution in the simulations [compare Figs. 3(b)-3(d) with Figs. 3(f)-3(h)]. Moreover, the spin configurations imprinted into the AFM at the edges of the disks do not vary significantly for these relatively small $H_{\mathrm{FC}}$. This explains why the loop asymmetry affects only the reversible part of the loops, since the vortex nucleation and annihilation are influenced mainly by the spin distribution at the disks edges. This new type of loop asymmetry is different from the ones in other exchange bias systems, where the asymmetry results from different irreversible magnetization mechanisms on either side of the loop [14-18]. Note that the possibility to controllably imprint different types of vortex states into the antiferromagnet evidences the local character of the exchange bias coupling.

When these structures are $\mathrm{FC}$ along $\theta_{\mathrm{FC}}=90^{\circ}$, the simulations generate a symmetric longitudinal hysteresis loop, with a nonzero transverse component [Fig. 2(c)]. The vortex core follows a curved trajectory, illustrated by the line in Fig. 3(h) and plotted in Fig. 2(f), corresponding to the nonzero transverse component. Figure $3(\mathrm{~h})$ shows that, for $\theta_{\mathrm{FC}}=90^{\circ}$, the only remanent moment is transverse, in

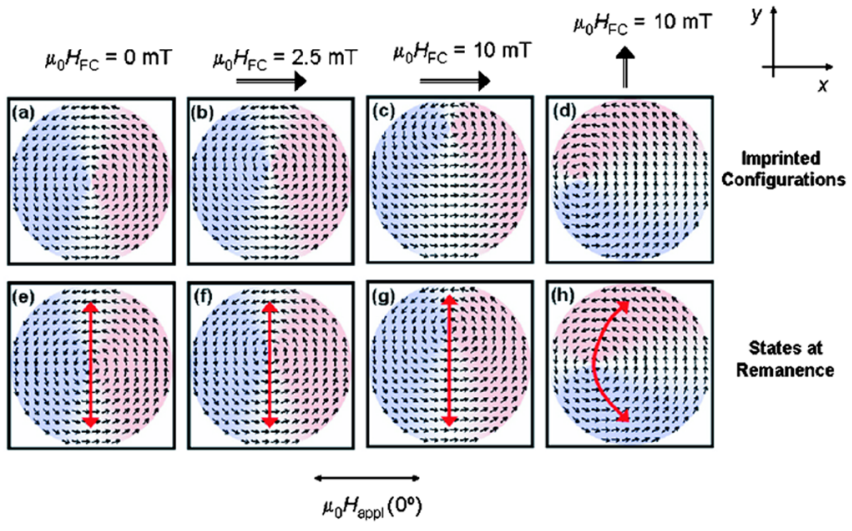

FIG. 3 (color online). Micromagnetic calculations of the spin configurations imprinted to the AFM when cooling in (a) zero field, (b) $\theta_{\mathrm{FC}}=0^{\circ}$ and $\mu_{0} H_{\mathrm{FC}}=2.5 \mathrm{mT}$, (c) $\theta_{\mathrm{FC}}=0^{\circ}$ and $\mu_{0} H_{\mathrm{FC}}=10 \mathrm{mT}$, and (d) $\theta_{\mathrm{FC}}=90^{\circ}$ and $\mu_{0} H_{\mathrm{FC}}=10 \mathrm{mT}$. (e) $-(\mathrm{h})$ are the spin configurations of the FM at remanence calculated for the imprinted configurations of (a)-(d), respectively. The shading represents the magnitude of the magnetization vector along the perpendicular to the field-cooling direction. The direction of the applied field during the hysteresis loops $\left(\mu_{0} H_{\text {appl }}\right)$ is also indicated. The lines illustrate the core trajectories. 


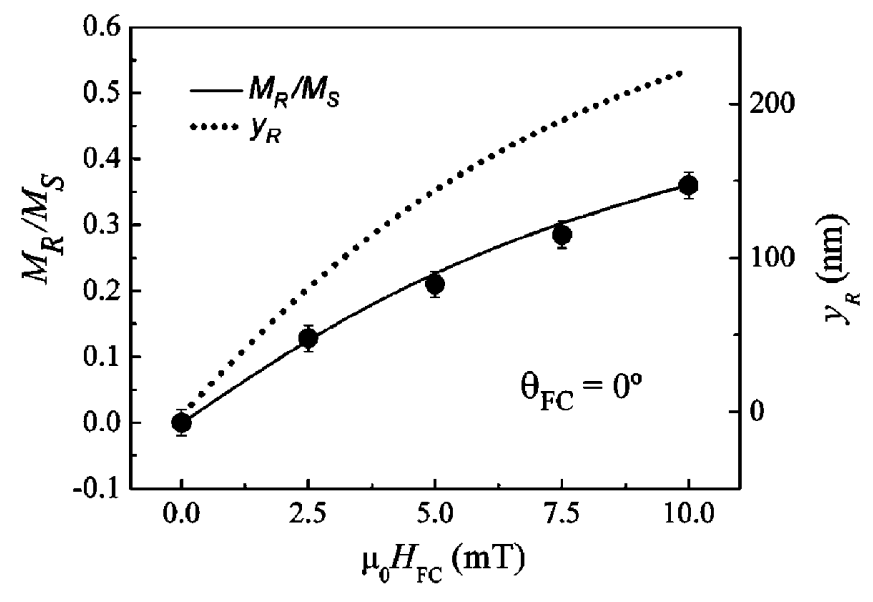

FIG. 4. Evolution of the measured (O) and calculated normalized remanent magnetization $M_{R} / M_{S}$ (continuous line) and the calculated core position at remanence $y_{R}$ (dotted line) from the FC disks $\left(\theta_{\mathrm{FC}}=0^{\circ}\right)$, as a function of cooling field $\mu_{0} H_{\mathrm{FC}}$.

agreement with the experimental results [Fig. 1(c)]. Note that the experimental loops shapes (Fig. 1) agree very well with the simulations (Fig. 2).

Further evidence for the straight and curved trajectories of the vortex core after FC the disks in $\mu_{0} H_{\mathrm{FC}}=10 \mathrm{mT}$ along $0^{\circ}$ and $90^{\circ}$ is also obtained by magnetic force microscopy imaging (see Ref. [32] for supplementary material). In particular, the imaging shows that, while the vortex core passes through the center of the disks after FC along $0^{\circ}$, a dipolar contrast stemming from the displaced vortex structure is always obtained after $\mathrm{FC}$ along $90^{\circ}$, where the vortex core never coincides with the disk center.

Finally, the dependence of the remanence to saturation ratio $M_{R} / M_{S}$ on $H_{\mathrm{FC}}$, for $\theta_{\mathrm{FC}}=0^{\circ}$, both from simulated and experimental data, are compared in Fig. 4. Excellent agreement between the theoretical and experimental values is obtained. As $H_{\mathrm{FC}}$ increases, $M_{R} / M_{S}$ also increases due to the progressive displacement of the imprinted vortex core with respect to the disk center during the cooling. Hence, by adjusting the vortex configuration imprinted to the antiferromagnet, it is possible to precisely control the loop asymmetry. The imprint of particular spin configurations into an AFM that a priori are not energetically favorable may assist in the future implementation of novel types of magnetoelectronic micro- and nanodevices.

Financial support from the NEXBIAS (No. HPRN-CT 2002-00296), the No. 2005SGR-00401 and the No. MAT2004-01679 research projects, and the Institut Català de Nanotecnologia (ICN) is acknowledged. Work at Argonne was supported by the U.S. Department of Energy, under Contract No. W-31-109-ENG-38. K. S. B. acknowledges support from NSERC Canada and G. S. A. and A. B. acknowledge funding from the NEXBIAS research network.

*Electronic address: jordi.sort@uab.es

†Electronic address: buchanan@anl.gov

[1] B. K. Tanner, Contemp. Phys. 20, 187 (1979).

[2] F. Nolting et al., Nature (London) 405, 767 (2000).

[3] P. G. Evans et al., Science 295, 1042 (2002).

[4] J. I. Martín et al., J. Magn. Magn. Mater. 256, 449 (2003).

[5] R. P. Cowburn et al., Phys. Rev. Lett. 83, 1042 (1999).

[6] J. K. Ha, R. Hertel, and J. Kirschner, Phys. Rev. B 67, 224432 (2003).

[7] K. Y. Guslienko et al., Phys. Rev. B 65, 024414 (2001).

[8] K. Yu. Guslienko et al., Phys. Rev. Lett. 96, 067205 (2006).

[9] M. Buess et al., Phys. Rev. Lett. 93, 077207 (2004).

[10] W. Kleemann, Int. J. Mod. Phys. B 7, 2469 (1993).

[11] J. Nogués et al., Phys. Rep. 422, 65 (2005).

[12] U. Nowak et al., Phys. Rev. B 66, 014430 (2002).

[13] J. C. S. Kools, IEEE Trans. Magn. 32, 3165 (1996).

[14] M. R. Fitzsimmons et al., Phys. Rev. Lett. 84, 3986 (2000).

[15] B. Beckmann, U. Nowak, and K. D. Usadel, Phys. Rev. Lett. 91, 187201 (2003).

[16] V. I. Nikitenko et al., Phys. Rev. Lett. 84, 765 (2000).

[17] J. Camarero et al., Phys. Rev. Lett. 95, 057204 (2005).

[18] J. Mejía-López, P. Soto, and D. Altbir, Phys. Rev. B 71, 104422 (2005).

[19] J. Nogués et al., Phys. Rev. B 61, R6455 (2000).

[20] D. Lederman, J. Nogués, and I. K. Schuller, Phys. Rev. B 56, 2332 (1997).

[21] D. Mauri et al., J. Appl. Phys. 62, 2929 (1987).

[22] J. Camarero et al., Phys. Rev. Lett. 91, 027201 (2003).

[23] C. Leighton et al., Phys. Rev. Lett. 84, 3466 (2000).

[24] S. Brück et al., Adv. Mater. 17, 2978 (2005).

[25] O. Petracic et al., Appl. Phys. Lett. 87, 222509 (2005).

[26] I. V. Roshchin et al., Europhys. Lett. 71, 297 (2005).

[27] J. Sort et al., Phys. Rev. Lett. 95, 067201 (2005).

[28] Z.-P. Li et al., Appl. Phys. Lett. 86, 072501 (2005).

[29] R. Nakatani et al., J. Magn. Magn. Mater. 286, 31 (2005).

[30] W. Jung et al., J. Appl. Phys. 97, 10K113 (2005).

[31] J. Sort et al., Appl. Phys. Lett. 88, 042502 (2006).

[32] See EPAPS Document No. E-PRLTAO-97-076632 for supplementary figures that show MFM images of Permalloy-IrMn disks obtained under different selected fields applied either parallel or perpendicular to the direction of the FC. For more information on EPAPS, see http:// www.aip.org/pubservs/epaps.html.

[33] LLG Micromagnetics Simulator ${ }^{\text {TM }}$, M. R. Scheinfein. 